
\title{
BReserch S Suare \\ Goji Berry Intake Increases Macular Pigment Optical Density in Healthy Adults: A Randomized Pilot Trial
}

\section{Xiang Li}

UC Davis

Roberta R. Holt

UC Davis

Carl L. Keen

UC Davis

\section{Lawrence S. Morse}

UC Davis Medical Center

Glenn Yiu

UC Davis Medical Center

Robert M. Hackman ( $\nabla$ rmhackman@ucdavis.edu )

UC Davis

\section{Research Article}

Keywords: Goji Berry, Macular Pigment, Optical Density, Healthy Adults

Posted Date: August 23rd, 2021

DOl: https://doi.org/10.21203/rs.3.rs-785757/v1

License: (c) (i) This work is licensed under a Creative Commons Attribution 4.0 International License.

Read Full License

Version of Record: A version of this preprint was published at Nutrients on December 9th, 2021. See the published version at https://doi.org/10.3390/nu13124409. 


\section{Abstract}

Age-related macular degeneration (AMD) is the third leading cause of blindness worldwide. Macular pigment optical density (MPOD), a biomarker for AMD, is a non-invasive measure to assess risk. The macula xanthophyll pigments lutein $(\mathrm{L})$ and zeaxanthin $(\mathrm{Z})$ protect against blue light and provide oxidant defense, which can be indexed by MPOD. This study examined the effects of L- and Z-rich goji berry intake on MPOD and skin carotenoids in healthy individuals. A randomized, unmasked, parallel-arm study was conducted with 27 participants, aged 45-65, who consumed either $28 \mathrm{~g}$ of goji berries or a supplement containing $6 \mathrm{mg} \mathrm{L}$ and $4 \mathrm{mg} \mathrm{Z} \mathrm{(LZ),} \mathrm{five} \mathrm{times} \mathrm{weekly} \mathrm{for} 90$ days. After 90 days, MPOD was significantly increased in the goji berry group at 0.25 and 1.75 retinal eccentricities; $R E ; p=0.029$ and $p=$ 0.044 , respectively), while no changes were noted in the LZ group. Skin carotenoids were significantly increased in the goji berry group at day $45(p=0.025)$ and day $90(p=0.006)$, but not in the LZ group. Regular intake of goji berries in a healthy middle-aged population increases MPOD, which may help prevent or delay the development of AMD.

\section{Introduction}

Age-related macular degeneration (AMD) is the leading cause of blindness among seniors in developed countries, and the third worldwide after cataract and glaucoma ${ }^{1,2}$. The disease is characterized by small to intermediate drusen with pigmentary changes in early stages but may progress rapidly in more advanced forms such as choroidal neovascularization or central geographic atrophy and eventually a loss of central vision ${ }^{3}$. Lutein (L), zeaxanthin (Z), and the isomer meso-zeaxanthin (meso-Z) are macular pigments that filter damaging blue light and provide oxidative defense in the macula ${ }^{3}$. These pigments are found in plants as xanthophylls and have been proposed to reduce the development and progression of $\mathrm{AMD}^{4}$. Assessment of the relative concentration of xanthophyll carotenoids in the retina can be measured non-invasively by psychophysical and objective methods, expressed as macular pigment optical density (MPOD) $)^{5}$. Numerous epidemiological studies report that individuals with a low MPOD level are at an increased risk of $\mathrm{AMD}^{6}$.

Dietary $L$ and $Z$ are found in certain fruits and vegetables with red, yellow, or orange color, egg yolk, and in some green leafy vegetables ${ }^{7,8}$. The dietary intake of $Z$ is lower than $L$ in all age groups and ethnicities in the U.S. ${ }^{9}$ Dietary intakes of $L$ and $Z$ are strongly associated with their serum levels, as well as with $M^{M P O D}{ }^{10}$. Previous studies have shown that plasma $Z$ and $L$, and MPOD can be increased by high intakes of these carotenoids from dietary sources or supplements ${ }^{11}$. Once the disease has progressed to intermediate AMD, dietary supplements are indicated, but no clinical evidence yet exists for interventions that can address the prevention of small-intermediate drusen with pigmentary changes, the initial clinical signs of macular disruption ${ }^{12}$.

Goji berry (Lycium barbarum L. and L. chinense), also termed wolfberry or Go Chi Zi, has been used in traditional Chinese medicine for more than 2,000 years $^{13}$. The bright red berry contains the highest 
amount of $Z$ among all known dietary sources ${ }^{14}$ and is mainly present in a dipalmitate form ${ }^{15}$. When zeaxanthin dipalmitate (ZD) was extracted from goji berries and compared to non-esterified $Z$, intake of the ZD complex showed a greater increase in plasma $Z$ in humans than the isolated form ${ }^{16}$. The berries also contain taurine, antioxidant vitamins, phenolic compounds, and select essential trace elements. A unique $L$. barbarum polysaccharide (LBP) fraction is also present that has shown anti-inflammatory and neuroprotective effects in animal and cell culture studies ${ }^{17}$.

The typical adult human eye has approximately 2.4 times more $Z$ than $L$ in the central fovea of the macula ${ }^{18}$, making goji berry intake a prime candidate for increasing MPOD. Nevertheless, the clinical evidence on goji berry and MPOD is scarce. One study investigating the effects of $25 \mathrm{~g}$ of daily consumption of goji berries in individuals with early AMD signs reported that both serum $Z$ and MPOD were significantly increased after 90 days ${ }^{19}$. However, this study had a broad age range (51 to 92 years of age), some participants smoked, and others had certain pre-existing medical conditions. Additionally, the authors only reported central MPOD values up to 0.5 retinal eccentricity (RE), whereas macular pathology and visual dysfunction in AMD may extend beyond that central region. Thus, measuring MPOD at various eccentricities over the entirety of the macula provides a more complete understanding of the impact of macular pigment on AMD.

In this study, we prospectively evaluated if consumption of $28 \mathrm{~g}$ of goji berries for 90 days could improve MPOD and skin carotenoid levels, an index of total carotenoid intake, among healthy middle-aged adults, 45 to 65 years old, compared with a commercially available supplement providing $6 \mathrm{mg}$ of $\mathrm{L}$ and $4 \mathrm{mg}$ of Z (LZ).

\section{Results}

Thirty-one healthy, middle-aged adult males and females (mean age of 56 years) with normal-appearing macula, were randomized into a prospective, parallel-arm, unmasked study between May 2019 and Jan 2020. The participants consumed either goji berries $(n=16)$ or the LZ supplement $(n=15)$ five days per week for 90 days. Twenty-eight individuals completed the intervention, while two in the goji berry and one from the LZ group were excluded from the data analysis due to measurement errors. Of the twenty-eight individuals who completed the intervention, data from one was subsequently removed after learning of a major change in dietary patterns that included a low intake of macronutrients between days 45 and 90 (Fig. 1).

Reported protocol compliance was greater than $96 \%$ for both groups, and no adverse symptoms were reported other than minor intestinal gas from one participant after goji berry intake. Table 1 presents the reported average intake of select nutrients in the habitual diet that may have affected eye health over the study period. No significant differences between groups were noted. The composition of the goji berries is presented in Table 2 . A daily goji berry serving provided $28.8 \mathrm{mg}$ of $Z$, which was substantially greater than the $4 \mathrm{mg}$ of $Z$ present in the supplement. Although sufficient extraction of $L$ from our goji berry samples could not be obtained, previous work by others estimated a $\mathrm{L}$ content of $0.15 \mathrm{mg}$ in $28 \mathrm{~g}$ of goji 
berries from six different goji berry samples collected in the Ningxia province of $\mathrm{China}^{20}$, the same region from which the goji berries used in this study were obtained.

Table 1

Mean intake of select dietary nutrients, apart from intake of goji berries (GB) or lutein and zeaxanthin (LZ) supplementation, collected once between day 0 and day 45, and again between day 45 and day 90 . Statistical analysis performed by two-tailed t test; values are the mean \pm S.E.M. or back transformed mean ${ }^{1}(95 \% \mathrm{Cl})$ of the log data obtained from ASA24. DHA: docosahexaenoic acid; DPA: docosapentaenoic acid; EPA: eicosapentaenoic acid.

\begin{tabular}{|c|c|c|c|}
\hline & GB & LZ & p value \\
\hline Energy (kcal) & $2146.4 \pm 187.7$ & $1984.3 \pm 151.5$ & 0.51 \\
\hline Protein (g) & $89.3 \pm 10.1$ & $72.7 \pm 7.2$ & 0.18 \\
\hline Total Fat (g) & $84.2 \pm 9.3$ & $84.4 \pm 8.8$ & 0.98 \\
\hline Carbohydrate (g) & $256 \pm 26$ & $241 \pm 17$ & 0.6 \\
\hline Vitamin A (mcg) & $807.8 \pm 120.6$ & $578.3 \pm 58.4$ & 0.07 \\
\hline Vitamin C (mg) & $120.0 \pm 18$ & $103.9 \pm 13.8$ & 0.48 \\
\hline Vitamin $E(m g)^{1}$ & $14.4(8.6,24.1)$ & $11.0(9.0,13.3)$ & 0.21 \\
\hline Zinc (mg) & $11.8 \pm 0.8$ & $9.5 \pm 0.9$ & 0.08 \\
\hline Retinol (mcg) & $307.1 \pm 51.5$ & $265.4 \pm 40.5$ & 0.52 \\
\hline$\beta$-carotene (mcg) & $5127.6 \pm 874.0$ & $3408.1 \pm 680.4$ & 0.13 \\
\hline a-carotene $(\mathrm{mcg})^{1}$ & $300.3(81.3,1109.0)$ & $205.9(87.7,483.4)$ & 0.58 \\
\hline$\beta$-cryptoxanthin (mcg) ${ }^{1}$ & $156.9(33.6,732.2)$ & $91.0(53.1,156.0)$ & 0.4 \\
\hline Lycopene (mg) ${ }^{1}$ & $7.2(3.1,15.0)$ & $3.6(1.7,7.3)$ & 0.2 \\
\hline Lutein + zeaxanthin $(\mathrm{mg})^{1}$ & $3.1(1.7,5.5)$ & $1.9(1.1,3.2)$ & 0.2 \\
\hline $\mathrm{DHA}(\mathrm{g})^{1}$ & $44.9(12.9,156.4)$ & $37.0(15.8,86.5)$ & 0.77 \\
\hline DPA $(g)^{1}$ & $16.2(7.1,36.6)$ & $9.3(5.1,16.9)$ & 0.23 \\
\hline $\operatorname{EPA}(\mathrm{g})^{1}$ & $11.8(2.5,56.5)$ & $13.5(6.0,30.2)$ & 0.86 \\
\hline
\end{tabular}


Table 2

Composition of select nutrients and carotenoids in $28 \mathrm{~g}$ of goji berries.

* Lutein estimated from goji berries

cultivated in Ningxia province, China (Zhao et al., 2013).

\begin{tabular}{|ll|}
\hline Nutrient & Amount \\
\hline Calorie $($ Kcal) & 95.1 \\
\hline Total Carbohydrate $(\mathrm{g})$ & 21.4 \\
\hline Fat $(\mathrm{g})$ & 0.4 \\
\hline Protein $(\mathrm{g})$ & 2.8 \\
\hline Fiber $(\mathrm{g})$ & 2.7 \\
\hline Total sugars $(\mathrm{g})$ & 15.1 \\
\hline Carotenoids & \\
\hline Zeaxanthin $(\mathrm{mg})$ & 28.8 \\
\hline$\beta$-carotene $(\mu \mathrm{g})$ & 225 \\
\hline Trans $\beta$-carotene $(\mu \mathrm{g})$ & 110 \\
\hline a-carotene $(\mu \mathrm{g})$ & 13.8 \\
\hline Lycopene $(\mu \mathrm{g})$ & $<5.6$ \\
\hline Lutein estimate* $(\mathrm{mg})$ & 0.15 \\
\hline
\end{tabular}

Baseline MPOD measures were similar between the goji berry and supplement groups (Table 3). No significant interaction effects for treatment and time were observed in any REs. A significant main effect of time was found for MPOD at $0.25 \mathrm{RE}(\mathrm{p}=0.023)$. In a sub-analysis, intake of goji berry, but not LZ, significantly increased MPOD at $0.25 \mathrm{RE}$ at day 90 compared to baseline ( $p=0.029 ; \mathrm{Fig}$. 2a). The main effect of time was also significant for MPOD at 1.75 RE ( $p=0.039)$. After the student t-test for pairwise comparisons, the MPOD at 1.75 RE was significantly increased at day 45 compared to the baseline $(p=$ $0.021)$, and to day 90 ( $p=0.044$; Fig. $2 b$ ). No significant MPOD changes were noted at any REs in the LZ group. 
Table 3

Baseline measurements of participants in the goji berry (GB) and the lutein and zeaxanthin supplement (LZ) group. Variables were not significantly different between the two groups. Statistical analysis was performed by two-tailed t-tests; data are presented as mean \pm S.E.M. MPOD: macular pigment optical density; RE: retinal eccentricity degrees; SC: skin carotenoid.

\begin{tabular}{|llll|}
\hline & GB group $(n=13)$ & LZ group $(n=14)$ & p value \\
\hline Age (years) & $55.9 \pm 1.7$ & $55.8 \pm 1.4$ & 0.94 \\
\hline Sex (F), $n(\%)$ & $9(69.2)$ & $10(71.4)$ & - \\
\hline MPOD & & & \\
\hline 0.25 RE & $0.67 \pm 0.06$ & $0.68 \pm 0.06$ & 0.88 \\
\hline 0.5 RE & $0.54 \pm 0.07$ & $0.59 \pm 0.05$ & 0.51 \\
\hline RE & $0.36 \pm 0.03$ & $0.40 \pm 0.03$ & 0.32 \\
\hline 1.75 RE & $0.16 \pm 0.02$ & $0.17 \pm 0.02$ & 0.77 \\
\hline SC Score & $369.5 \pm 44.9$ & $397.8 \pm 39.6$ & 0.64 \\
\hline
\end{tabular}

Baseline skin carotenoid scores were not significantly different between the goji berry and LZ groups (Table 3). No significant interaction effects for treatment and time were observed. However, the main effect of time was significant $(p=0.011)$. This corresponded to a significant increase from baseline after 45 and 90 days in the goji berry group ( $p=0.025$ and 0.006 , respectively), while no significant changes were noted in the LZ group (Fig. 3).

Overall, skin carotenoid scores were significantly correlated with MPOD at $0.25(\rho=0.33, p=0.004), 0.5$ $(\rho=0.41, p=0.0002)$, and $1 \operatorname{RE}(\rho=0.38, p=0.0007$; supplementary Fig. 1.1$)$. Skin carotenoid score was not correlated with MPOD at any of the REs for the goji berry group (supplementary Fig. 1.2). In contrast, for the LZ group, the skin carotenoid score was significantly correlated with MPOD at $0.25,0.5$, and 1 REs (0.25 RE: $\rho=0.55, p=0.0003 ; 0.5$ RE: $\rho=0.57, p=0.0002 ; 1$ RE: $\rho=0.54, p=0.0004$ ), with a trend at 1.75 $\operatorname{RE}(\rho=0.31, p=0.06$; supplementary Fig. 1.3).

\section{Discussion}

The optical biomarker, MPOD, was significantly increased in healthy adults at 0.25 and 1.75 REs in the goji berry group after 90 days of intake. This is consistent with a report that goji berry intake increased MPOD in a Chinese population at-risk for intermediate $\mathrm{AMD}^{19}$. Our results are similar to another study that reported consumption of a milk-based supplement containing $10 \mathrm{mg} / \mathrm{d}$ of $Z$ derived from goji berries for 90 days protected against macular hypopigmentation and drusen development in a population of generally healthy males and females (65 to 70 years of age $)^{21}$. Interestingly, we observed a significant increase in MPOD at $1.75 \mathrm{RE}$, but not at 0.5 or $1 \mathrm{RE}$, in the goji berry group. A possible explanation for this 
trend is the relatively low macular pigment at $1.75 \mathrm{RE}$ compared to the other REs that may increase the potential for improved MPOD in this peripheral area of the macula.

Our results suggest that higher intake of $Z$ relative to $L$ may increase MPOD. This is consistent with a four-month intervention in healthy young adults (18 to 32 years of age) reporting significantly increased MPOD levels after daily intake of a supplement containing $20 \mathrm{mg} \mathrm{Z}$, or $26 \mathrm{mg} \mathrm{Z}$ with $8 \mathrm{mg} \mathrm{L}$ plus $190 \mathrm{mg}$ of mixed omega-3 fatty acids ${ }^{22}$. Our findings are also consistent with data from 11 randomized controlled trials where supplementation with at least $10 \mathrm{mg}$ of the macular carotenoids is effective at increasing MPOD ${ }^{23}$.

Significant correlations were observed between the overall skin carotenoid score and MPOD, which is consistent with clinical results of carotenoid supplementation ${ }^{24}$. Further analysis demonstrated that $L Z$, but not goji berry intake, was significantly influencing this trend. Previous work has shown that the total carotenoid content in skin and blood is associated with macular pigment carotenoid accumulation ${ }^{24}$. In the current trial, goji berry intake was significantly associated with the skin carotenoid score, which is consistent with the recent observation by Obana et al. who reported a significant increase in skin carotenoid levels following the intake of $20 \mathrm{mg}$ of $\mathrm{L}$ and $4 \mathrm{mg}$ of $Z$ along with modest amounts of vitamins and minerals for 16 weeks ${ }^{25}$. Improvements in macular pigment optical volume and MPOD for those with a $\mathrm{BMI}<25 \mathrm{~kg} / \mathrm{m}^{2}$ were also reported ${ }^{25}$. We did not collect BMI data in the current trial, so we are unable to assess the impact of BMI on MPOD and skin carotenoid score. The skin photometer detects overall carotenoid content, and since goji berries are also rich in $\beta$-carotene, neoxanthin, and cryptoxanthin ${ }^{26}$, these carotenoids likely influenced the skin measurements, which would not reflect the selective carotenoid accumulation of $L$ and $Z$ in the macula. Other components in goji berries may also have influenced the significant increase in MPOD, since the berries contain taurine, vitamin C, zinc, and LBP, which may lower oxidant stress and improve eye health ${ }^{27-29}$. Studies on animals and cell lines suggest that bioactive compounds in goji berries, such as LBP, can protect against AMD by reducing oxidative stress and cell apoptosis in retinal pigment epithelium ${ }^{30}$. Taken together, it is reasonable that MPOD may not correlate with skin carotenoids in the goji berry group.

To our knowledge, the impact of goji berry intake on MPOD in healthy middle-aged people has not been previously reported. While others have noted improved MPOD after LZ supplementation among people with low MPOD baseline levels ${ }^{31}$, our findings suggest that even in populations with high normal MPOD values, a significant increase can be detected after goji berry consumption at the most central part of the macula ( $0.25 \mathrm{RE})$. A meta-analysis regarding the effects of $L, Z$, and meso-Z supplementation noted that the MPOD at baseline was inversely associated with macular responses, suggesting individuals with a relatively lower macular pigment status may receive more benefit with higher amounts of $L$ or $Z^{32}$.

The Age-Related Eye Disease Study 2 (AREDS2) trial assessed the impact of dietary supplements containing $10 \mathrm{mg}$ of L, $2 \mathrm{mg}$ of Z, $500 \mathrm{mg}$ of vitamin C, $400 \mathrm{IU}$ of vitamin E, 80 or $25 \mathrm{mg}$ of zinc, $2 \mathrm{mg}$ of copper, and/or $350 \mathrm{mg}$ of docosahexaenoic acid plus $650 \mathrm{mg}$ of eicosapentaenoic acid ${ }^{33}$. The results 
showed a significantly reduced rate of progression from intermediate- to late-stage AMD after five years $^{34,35}$. Secondary analyses of the study indicated protective roles of $L$ and $Z^{33}$. We did not use the AREDS2 supplement for the comparison group because this formula has only been shown effective for those with intermediate $A M D^{35}$, and no clinical evidence exists for its efficacy in our study population of healthy people. In addition, we note that $80 \mathrm{mg}$ of zinc in the AREDS2 supplement is twice the upper limit of recommended daily intakes for zinc.

In epidemiological studies, $L$ and $Z$ intakes have been inversely associated with development of $A M D^{32,36}$. The reported dietary intake of $L$ plus $Z$, not including the berries or supplement. was 3.1 and 1.9 $\mathrm{mg} / \mathrm{d}$ in the goji berry and supplement groups, respectively, which is higher than the typical estimated intakes in the US of $1.58-1.76 \mathrm{mg} / \mathrm{d}^{37}$. Three to five $\mathrm{mg} / \mathrm{d}$ of $\mathrm{L}$ and $\mathrm{Z}$ have been recommended to help support normal macular function ${ }^{8}$, although no Recommended Dietary Allowance values yet exist. Few studies have explored the effects of $L$ and $Z$ from a whole food on MPOD. Daily consumption of one Hass avocado containing $0.5 \mathrm{mg}$ of $L$ over six months was associated with a significant increase in MPOD in healthy adults ${ }^{38}$. In contrast, no increase in MPOD was observed after consuming one Hass avocado daily for three months ${ }^{39}$. Daily consumption of egg yolks providing $1.38 \mathrm{mg} \mathrm{L}$ and $0.21 \mathrm{mg} \mathrm{Z}$ resulted in a significant increase in MPOD and other measures of visual acuity in older adults with signs of early-stage AMD after 12 months ${ }^{40}$. Another study giving older adults two egg yolks/day for five weeks, followed by four egg yolks/day for five weeks reported increases in MPOD, in some subjects but only among those with low MPOD values at the start of the intervention ${ }^{41}$.

Our study has some limitations. Choice of a control is always a challenge in whole food studies, since masking is an issue. A commercially available LZ supplement was used, rather than an inert capsule, since our research design was intended to compare options available to consumers and explore the role of goji berries over and above the intake of purified $L$ and $Z$. Volunteers were not screened for low MPOD as an inclusion criteria. Finally, although MPOD was the primary outcome measure in this study, other ocular measurements such as contrast sensitivity and best corrected visual acuity were not assessed. Future studies on goji berry intake and eye health should combine functional and anatomic measurements.

In conclusion, this study showed that 90 days of goji berry consumption was associated with an increase in MPOD in middle-aged healthy adults. In addition to $L$ and $Z$, other bioactive compounds in goji berries may be involved in the increase in MPOD. Further research on goji berries is warranted as both a dietary strategy to help reduce the risk of AMD or to serve as part of an integrative approach to mitigate the consequences of this disorder.

\section{Methods}

\section{Participants}


Eighty-eight volunteers, ages from 45 to 65 years old, were recruited from an online website and public advertisements in the greater Sacramento, California area. Participants provided informed consent and were screened with a questionnaire. Inclusion criteria were being generally healthy (not currently under medical supervision, free from self-reported diabetes, cancer, heart, kidney or liver diseases and gastrointestinal disorders), having a normal macular condition as verified by an optometrist, and if relevant, prescribed the same medication regime for at least six months that was not related to carotenoid metabolism and approved by the study physician. Exclusion criteria were a dislike of, or allergy to goji berries, diseases of the eye, malabsorption problems, substance or alcohol abuse, smoking, drugs for management of lipids, glucose or blood pressure, dietary supplements other than multivitamins and minerals that provided greater than $100 \%$ of the U.S. Dietary Reference Intake, or any supplement containing $L$ or $Z$. The intervention was registered on ClinicalTrials.gov (NCT03983525), with the first posted date of 06/12/2019, complied with the tenets of the Declaration of Helsinki, was approved by the Institutional Review Board of the University of California, Davis and was conducted at the UC Davis Ragle Human Nutrition Research Center.

\section{Study design}

Qualified participants were randomized into a prospective, parallel-arm, unmasked study to consume either $28 \mathrm{~g}$ of goji berries or a commercially available supplement of $L$ and $Z$ five days per week for 90 days. Study measurements were collected at baseline (prior to supplement or goji berry intake; day 0 ), and $45 \pm 2$ days and $90 \pm 2$ days after intake.

Twenty-eight grams of goji berries is considered a single serving size $\mathrm{H}^{42}$. We used USDA-certified organic goji berries grown in the Ningxia region of northern China and provided by Navitas Organics, Novato CA, USA. The goji berries were portioned into clean, single-serving plastic bags and provided in 45-day allotments. The commercially available supplements (Source Naturals, Scotts Valley CA, USA) contained $6 \mathrm{mg}$ of $\mathrm{L}$ and $4 \mathrm{mg}$ of $Z$ per serving and were repackaged into 45 -day supplies in clean plastic bottles. Compliance was monitored by a self-administered log. Habitual dietary information was collected with the Automated Self-Administered 24-hour dietary assessment web-based tool (ASA24; https://epi.grants.cancer.gov/asa24) once between day 0 and 45 , and once again between day 45 and 90.

The MPOD was assessed by the psychophysical method of customized heterochromatic flicker photometry using a macular densitometer (Macular Metrics, Providence, RI, USA). After participants viewed a five-minute video detailing the measurement procedures, they were dark-adapted for seven minutes and then began the test. The light intensity of each relevant wavelength was calibrated with a photodiode. The flicker frequency was selected based on a preliminary test of the participant's sensitivity. The task was to eliminate or minimize the flicker in the visual field three times by turning a dial that changed the intensity of a 460-nm light. Each participant performed the test while looking directly at the flickering light at $0.25,0.5,1$, and 1.75 RE degrees, representing the MPOD level from the center to the periphery of the macula. 
Skin carotenoid content was measured by reflection spectroscopy ("Veggie Meter", Longevity Link Corporation, Salt Lake City, UT, USA). After cleaning, the tip of the right index finger was inserted into the device and three measurements were collected. A skin carotenoid score was calculated by the system software. Carotenoids that exist in human plasma, including $\beta$-carotene, lycopene, $L, Z$, and their isomers have been successfully detected in toto and quantified by this device ${ }^{43,44}$, which has been validated to reflect fruit and vegetable consumption ${ }^{45}$.

\section{Statistical analysis}

Sample size was based on a study that assessed the impact of a Z supplement on MPOD in 24 healthy people ${ }^{46}$. Statistical analyses were performed with JMP version 16 (SAS Institute Inc., Cary, NC, USA). Two-tailed t-tests evaluated potential between-group differences at baseline. The MPOD and skin carotenoid data were analyzed with mixed-effects models using time and treatment as the main factors, with age and sex as the covariates, and participant ID as the random effect. For significant main effects, student t-tests determined significant within groups pairs. $P$ values of 0.05 or less were considered statistically significant. Correlation coefficients between the outcome measures were determined via Spearman's method. Results are presented for illustration purposes as mean \pm S.E.M. The mean values of the dietary intake data were compared by two-tailed t-tests, which were log-transformed when necessary, and presented as the mean \pm S.E.M. or the back-transformed mean with $95 \%$ confidence intervals $(\mathrm{Cl})$.

\section{Declarations}

\section{Acknowledgements}

We thank Prof. John Werner and Ms. Susan Garcia, UC Davis Department of Ophthalmology and Vision Science, for their technical and scholarly support. We thank Navitas Organics, Novato, CA for the goji berries used in this study.

\section{Author contributions}

X.L. conceptualized the study, contributed to the study design, conducted the intervention, performed the data analyses, drafted the original manuscript.

R.R.H. contributed to data curation and interpretation.

C.L.K. contributed to data interpretation.

L.S.M. contributed to the study design and served as the study physician.

G.Y. contributed to data interpretation.

R.M.H. contributed to conceptualization, the study design, and project administration. 


\section{Additional information}

\section{Competing interests}

This study was supported in part from UC Davis graduate student awards to X.L. The authors declare no competing interests.

\section{References}

1. Heesterbeek, T. J., Lorés-Motta, L., Hoyng, C. B. \& Lechanteur, Y. T. E. \& den Hollander, A. I. Risk factors for progression of age-related macular degeneration. Ophthalmic Physiol. Opt, 40, 140-170 (2020).

2. World Health Organization. Blindness and vision impairment WHO https://www.who.int/newsroom/fact-sheets/detail/blindness-and-visual-impairment (2018).

3. Mitchell, P., Liew, G., Gopinath, B. \& Wong, T. Y. Age-related macular degeneration., 392, 1147-1159 (2018).

4. Eisenhauer, B., Natoli, S., Liew, G. \& Flood, V. M. Lutein and zeaxanthin - Food sources, bioavailability and dietary variety in age-related macular degeneration protection. Nutrients, 9, 120 (2017).

5. Howells, O., Eperjesi, F. \& Bartlett, H. Measuring macular pigment optical density in vivo: A review of techniques. Graefe's Arch. Clin. Exp. Ophthalmol, 249, 315-347 (2011).

6. Arunkumar, R., Calvo, C. M., Conrady, C. D. \& Bernstein, P. S. What do we know about the macular pigment in AMD: The past, the present, and the future. Eye, 32, 992-1004 (2018).

7. Rodriguez-Concepcion, M. et al. A global perspective on carotenoids: Metabolism, biotechnology, and benefits for nutrition and health. Prog. Lipid Res, 70, 62-93 (2018).

8. Ranard, K. M. et al. Dietary guidance for lutein: consideration for intake recommendations is scientifically supported. Eur. J. Nutr, 56, 37-42 (2017).

9. Johnson, E. J., Maras, J. E., Rasmussen, H. M. \& Tucker, K. L. Intake of lutein and zeaxanthin differ with age, sex, and ethnicity. J. Am. Diet. Assoc, 110, 1357-1362 (2010).

10. Mares, J. A. et al. Predictors of optical density of lutein and zeaxanthin in retinas of older women in the Carotenoids in Age-Related Eye Disease Study, an ancillary study of the Women's Health Initiative. Am. J. Clin. Nutr, 84, 1107-1122 (2006).

11. Carpentier, S., Knaus, M. \& Suh, M. Associations between lutein, zeaxanthin, and age-related macular degeneration: An overview. Crit. Rev. Food Sci. Nutr, 49, 313-326 (2009).

12. Hernández-Zimbrón, L. F. et al. Age-Related Macular Degeneration: New Paradigms for Treatment and Management of AMD. Oxid. Med. Cell. Longev. 2018, (2018). 
13. Potterat, O. Goji (Lycium barbarum and L. chinense): Phytochemistry, pharmacology and safety in the perspective of traditional uses and recent popularity. Planta Med, 76, 7-19 (2010).

14. Widomska, J., Paul Sangiovanni, J. \& Subczynski, W. K. Why is zeaxanthin the most concentrated xanthophyll in the central fovea? Nutrients 12,1-25(2020).

15. Karioti, A., Bergonzi, M. C., Vincieri, F. F. \& Bilia, A. R. Validated method for the analysis of Goji berry, a rich source of Zeaxanthin dipalmitate. J. Agric. Food Chem, 62, 12529-12535 (2014).

16. Breithaupt, D. E., Weller, P., Wolters, M. \& Hahn, A. Comparison of plasma responses in human subjects after the ingestion of 3R,3R'-zeaxanthin dipalmitate from wolfberry (Lycium barbarum) and non-esterified 3R,3R'-zeaxanthin using chiral high-performance liquid chromatography. Br. J. Nutr, 91, 707-713 (2004).

17. Amagase, H. \& Farnsworth, N. R. A review of botanical characteristics, phytochemistry, clinical relevance in efficacy and safety of Lycium barbarum fruit (Goji). Food Res. Int, 44, 1702-1717 (2011).

18. Bone, R. A., Landrum, J. T., Fernandez, L. \& Tarsis, S. L. Analysis of the macular pigment by HPLC: Retinal distribution and age study. Investig. Ophthalmol. Vis. Sci, 29, 843-849 (1988).

19. Li, S. et al. Macular pigment and serum zeaxanthin levels with Goji berry supplement in early agerelated macular degeneration. Int. J. Ophthalmol, 11, 970-975 (2018).

20. Zhao, L. Q., Qiu, Z. Q., Narasimhamoorthy, B. \& Greaves, J. A. Development of a rapid, highthroughput method for quantification of zeaxanthin in Chinese wolfberry using HPLC-DAD. Ind. Crops Prod, 47, 51-57 (2013).

21. Bucheli, P. et al. Goji berry effects on macular characteristics and plasma antioxidant levels. Optom. Vis. Sci, 88, 257-262 (2011).

22. Bovier, E. R., Renzi, L. M. \& Hammond, B. R. A double-blind, placebo-controlled study on the effects of lutein and zeaxanthin on neural processing speed and efficiency. PLoS One, 9, 1-6 (2014).

23. Ma, L. et al. Lutein, zeaxanthin and meso-zeaxanthin supplementation associated with macular pigment optical density. Nutrients, 8, 426 (2016).

24. Conrady, C. D. et al. Correlations between macular, skin, and serum carotenoids. Investig. Ophthalmol. Vis. Sci, 58, 3616-3627 (2017).

25. Obana, A. et al. Effect of an antioxidant supplement containing high dose lutein and zeaxanthin on macular pigment and skin carotenoid levels. Sci. Rep, 10, 1-12 (2020).

26. Wang, C. C., Chang, S. C., Inbaraj, B. S. \& Chen, B. H. Isolation of carotenoids, flavonoids and polysaccharides from Lycium barbarum $L$. and evaluation of antioxidant activity. Food Chem, 120, 184-192 (2010).

27. Song, M. K., Salam, N. K., Roufogalis, B. D. \& Huang, T. H. W. Lycium barbarum (Goji Berry) extracts and its taurine component inhibit PPAR-y-dependent gene transcription in human retinal pigment epithelial cells: Possible implications for diabetic retinopathy treatment. Biochem. Pharmacol, 82, 1209-1218 (2011). 
28. Yossa Nzeuwa, I. B. et al. Comparative Metabolic Profiling of Lycium Fruits (Lycium barbarum and Lycium chinense) from Different Areas in China and from Nepal. J. Food Qual. 2019, (2019).

29. Bungau, S. et al. Health Benefits of Polyphenols and Carotenoids in Age-Related Eye Diseases. Oxid. Med. Cell. Longev. 2019, (2019).

30. Neelam, K., Dey, S., Sim, R., Lee, J. \& Au Eong, K. G. Fructus lycii: A natural dietary supplement for amelioration of retinal diseases. Nutrients, 13, 246 (2021).

31. Trieschmann, M. et al. Changes in macular pigment optical density and serum concentrations of its constituent carotenoids following supplemental lutein and zeaxanthin: The LUNA study. Exp. Eye Res, 84, 718-728 (2007).

32. Ma, L. et al. Lutein and zeaxanthin intake and the risk of age-related macular degeneration: A systematic review and meta-analysis. Br. J. Nutr, 107, 350-359 (2012).

33. Chew, E. Y. et al. Secondary analyses of the effects of lutein/zeaxanthin on age-related macular degeneration progression AREDS2 report no. 3. JAMA Ophthalmol, 132, 142-149 (2014).

34. Kassoff, A. et al. A randomized, placebo-controlled, clinical trial of high-dose supplementation with vitamins $\mathrm{C}$ and $\mathrm{E}$, beta carotene, and zinc for age-related macular degeneration and vision loss: AREDS report no. 8. Arch. Ophthalmol, 119, 1417-1436 (2001).

35. Chew, E. Y. et al. Lutein + zeaxanthin and omega-3 fatty acids for age-related macular degeneration: The Age-Related Eye Disease Study 2 (AREDS2) randomized clinical trial. JAMA - J. Am. Med. Assoc, 309, 2005-2015 (2013).

36. Chiu, C. J. et al. The relationship of major american dietary patterns to age-related macular degeneration. Am. J. Ophthalmol, 158, 118-127 (2014).

37. National Health and Nutrition Exmaination Survey. What We Eat in America, Nutrient Intakes from Food and Beverages, NHANES 2013-2014 USDA https://www.ars.usda.gov/nea/bhnrc/fsrg (2014).

38. Scott, T. M., Rasmussen, H. M., Chen, O. \& Johnson, E. J. Avocado consumption increases macular pigment density in older adults: A randomized, controlled trial. Nutrients, 9, 919 (2017).

39. Edwards, C. G. et al. Effects of 12-week avocado consumption on cognitive function among adults with overweight and obesity. Int. J. Psychophysiol, 148, 13-24 (2020).

40. Van Der Made, S. M., Kelly, E. R., Kijlstra, A., Plat, J. \& Berendschot, T. T. J. M. Increased Macular Pigment Optical Density and Visual Acuity following Consumption of a Buttermilk Drink Containing Lutein-Enriched Egg Yolks: A Randomized, Double-Blind, Placebo-Controlled Trial. J. Ophthalmol. 2016, (2016).

41. Vishwanathan, R., Goodrow-Kotyla, E. F., Wooten, B. R., Wilson, T. A. \& Nicolosi, R. J. Consumption of 2 and 4 egg yolks/d for 5 wk increases macular pigment concentrations in older adults with low macular pigment taking cholesterol-lowering statins. Am. J. Clin. Nutr, 90, 1272-1279 (2009).

42. USDA. FoodData Central Goji Berries, Dried USDA https://fdc.nal.usda.gov/fdc-app.html\#/fooddetails/173032/nutrients (2018). 
43. Jahns, L. et al. Concurrent validity of skin carotenoid status as a concentration biomarker of vegetable and fruit intake compared to multiple 24-h recalls and plasma carotenoid concentrations across one year: a cohort study. Nutr. J, 18, 1-8 (2019).

44. Rush, E., Amoah, I., Diep, T. \& Jalili-Moghaddam, S. Determinants and suitability of carotenoid reflection score as a measure of carotenoid status. Nutrients, 12, 113 (2020).

45. Pitts, S. B. J. et al. A non-invasive assessment of skin carotenoid status through reflection spectroscopy is a feasible, reliable and potentially valid measure of fruit and vegetable consumption in a diverse community sample. Public Health Nutr, 21, 1664-1670 (2018).

46. Iannaccone, A., Carboni, G., Forma, G., Mutolo, M. \& Jennings, B. Macular Pigment Optical Density and Measures of Macular Function: Test-Retest Variability, Cross-Sectional Correlations, and Findings from the Zeaxanthin Pilot Study of Response to Supplementation (ZEASTRESS-Pilot). Foods, 5, 32 (2016).

\section{Figures}




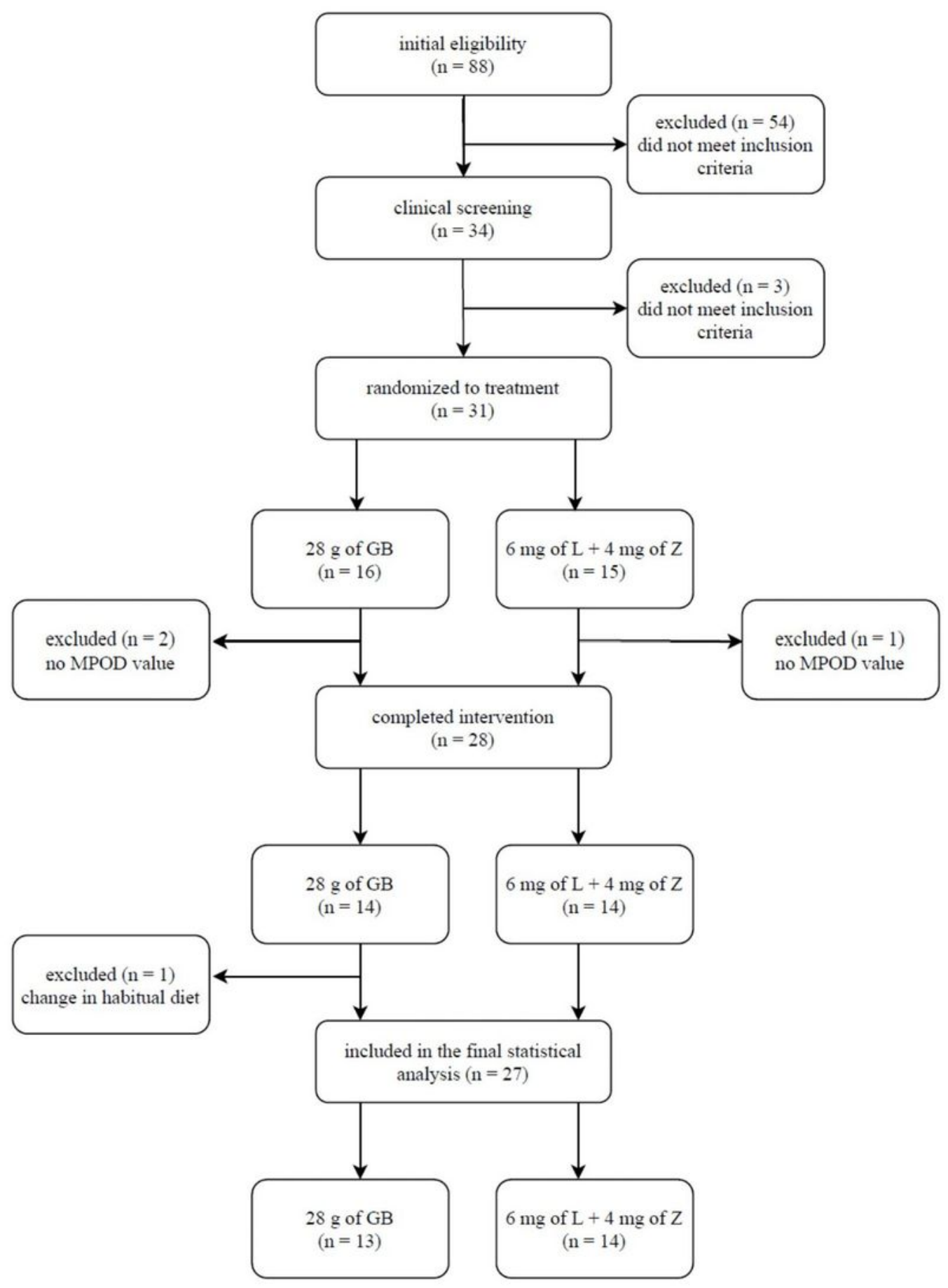

\section{Figure 1}

Participant flow diagram. Thirty-one participants were randomly assigned to consume either $28 \mathrm{~g}$ of goji berries (GB) or a supplement containing $6 \mathrm{mg}$ of lutein (L) and $4 \mathrm{mg}$ of zeaxanthin (Z), five times per week for 90 days. Twenty-eight individuals completed the study. An n=13 in the GB group and an n=14 in the LZ group were used in the statistical analysis. 


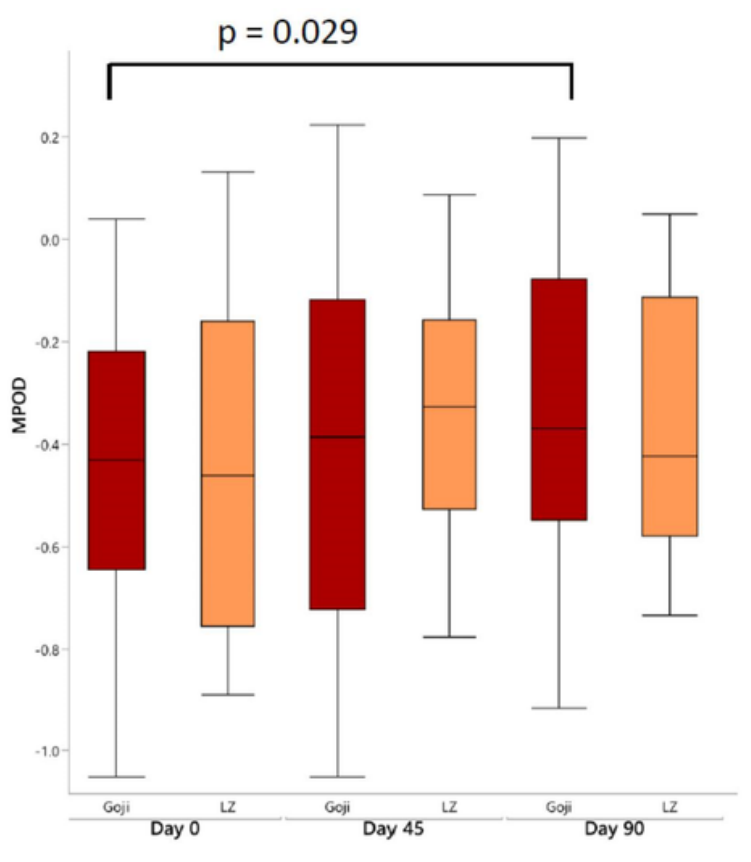

a

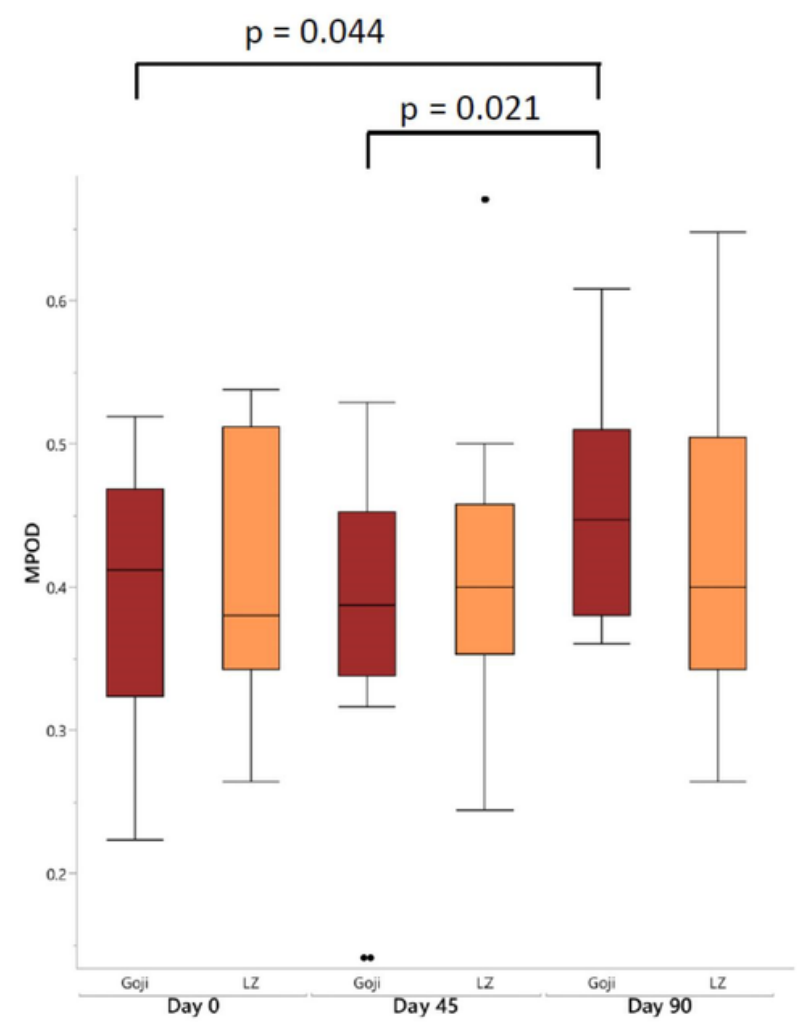

b

Figure 2

2a. Three months of goji berry intake increased macular pigment optical density (MPOD) at 0.25 retinal eccentricity (RE) degrees, at Day 90 compared to baseline (Day 0). Statistical analysis performed by mixed models using time and treatment as the main factors, and age and sex as the covariates with $t$ student t-test for pairwise comparisons; data shown for illustration purposes as mean \pm S.E.M. $2 b$. Three months of goji berry intake increased macular pigment optical density (MPOD) at 1.75 retinal eccentricity (RE) degrees, at Day 90 compared to baseline and at day 45. Statistical analysis performed by mixed models using time and treatment as the main factors, and age and sex as the covariates with student ttest for pairwise comparisons; data shown for illustration purposes as mean \pm S.E.M. 


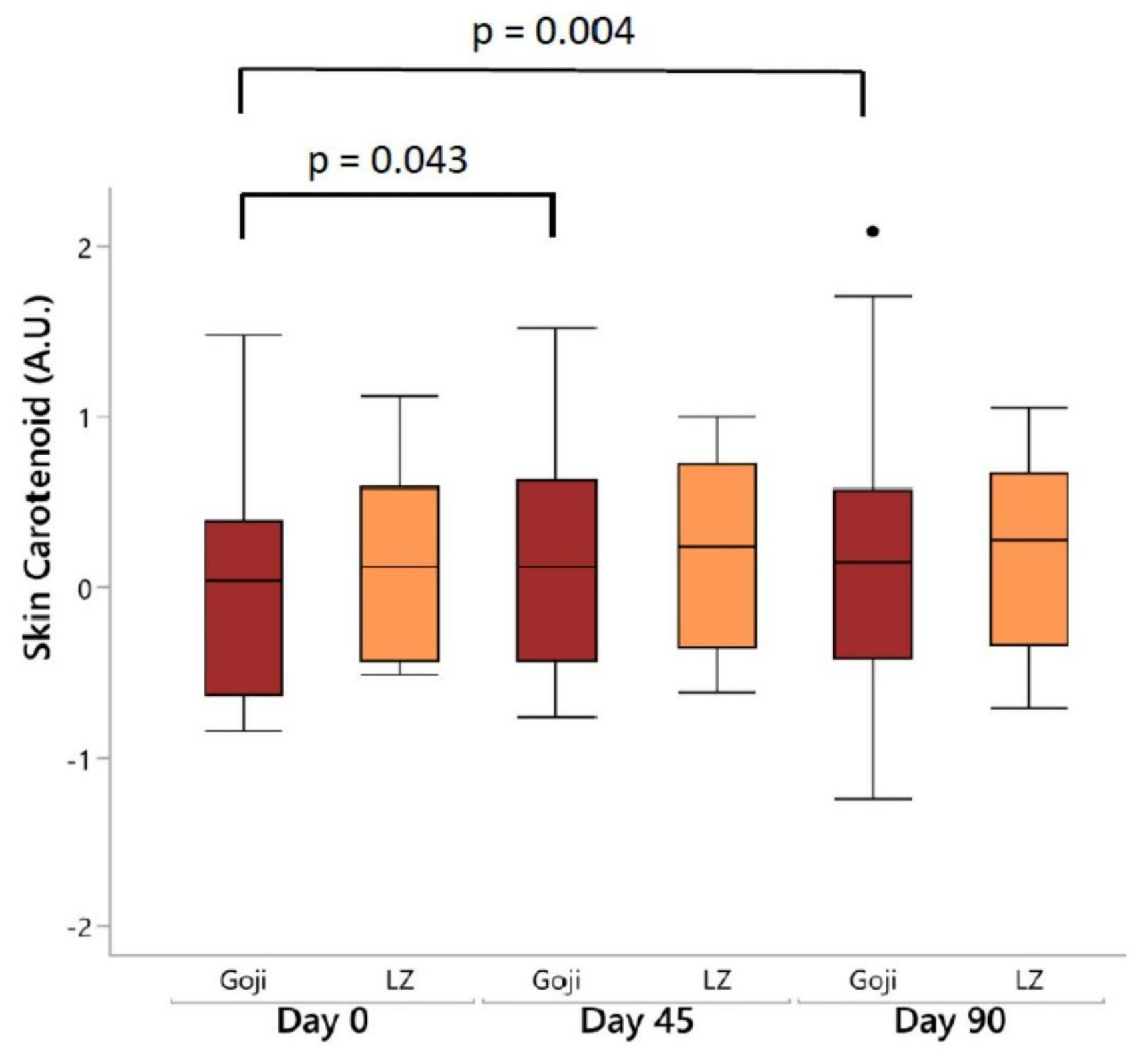

Figure 3

Three months of goji berry intake increased skin carotenoid scores at Day 45 and Day 90 compared to Day 0. No changes in the lutein and zeaxanthin supplement (LZ) group were noted. Statistical analysis performed by mixed models using time and treatment as the main factors, and age and sex as the covariates with student t-test for pairwise comparisons; data shown for illustration purposes as mean \pm S.E.M.

\section{Supplementary Files}


This is a list of supplementary files associated with this preprint. Click to download.

- Gojiberrysubmissionsupplementaryinfofigure1.11.21.3.pdf 